\title{
TWIST2 EXPRESSIONS IN PATIENTS WITH SQUAMOUS CELL CARCINOMA OF THE URINARY BLADDER SHOWED DOWN REGULATION CORRELATED WITH GOOD SURVIVAL AND LOW INCIDENCE OF DISTANT METASTASIS
}

By

\author{
MOHAMED WISHAHI ${ }^{1 *}$, HEBA KHALIL ${ }^{2}$ and NOURA KAMEL ${ }^{3}$ \\ Department of Urology ${ }^{1}$, Department of Pathology ${ }^{2}$, Theodor Bilharz Research \\ Institute, Imbaba P.O.B. 30, Giza, and Department of Pathology ${ }^{2}$, National Research \\ Center, Cairo, Egypt. ( ${ }^{\star}$ Correspondence: Email: moh.weshahy@gmail.com)
}

\begin{abstract}
This study evaluated the association between expression of Twist 2 monogenic, with pathological features and clinical outcomes of squamous cell carcinoma of the urinary bladder (SCCUB) following radical cystectomy (RC). Immunohistochemical staining Twist 2 was performed on tissue archival samples comprising normal urothelium from ten controls, cystectomy specimens from 87 patients with T2 N0 M0 and T3-4a N0 M0 of bladder squamous cell carcinoma (SCC).all patients with T2-4 SCC undergone radical cystectomy and urinary diversion, follow up was for 5-10 years to assess overall survival (OS) and disease free survival (DFS). Specimens from 10 muscle-invasive urothelial carcinoma patients were reassessed for Twist 2 expression to represent over- expression of Twist2. All ten controls had normal status of biomarkers which was negative. Negative or, low, and medium expression of Twist2 was noted in SCC of the urinary bladder of local pathological status with no lymph node metastasis or distant metastasis, clinicopathologic characteristics of the cohort were pT2N0 M0 (n=659 and pT3-4a N0 M0 $(n=22)$. Twist 2 negative expression was in 36 case, weak expression $(n=33)$, medium expression $(\mathrm{n}=16)$, there were no strong expression. Twist 2 low-regulation with combination of tomor stage in SCC of the urinary bladder werel independently associated with overall survival and free disease survival, 7.7 and 5 years survival in pT2 N0 M0 $(n=65)$ were $89 \%$ and $13 \%$ respectively, 7.7 and 5 years survival in pT3-4a N0 M0(n=22) were $72.7 \%$ and $22.7 \%$ respectively,
\end{abstract}

Key-Words: Twist2; Squamous cell carcinoma, urinary bladder: Epithelial-mesenchymal transition; metastasis; Prognostic biomarker

\section{Introduction}

Bladder cancer is one of the most common cancers worldwide. Numerous factors, including genetic poly-morphisms and genetic and epigenetic alterations, are involved in the tumorigenesis, progression, and metastasis of bladder cancer (Li et al, 2016). Most cases are of transitional histology, despite good prognosis for non-muscle invasive urothelial carcinoma patients, recurrence is common, and up to $30 \%$ of recurrences progress to a muscle-invasive condition Approximately $50 \%$ of the patients with muscle-invasive cancer, including those who progress to this state from non-muscle invasive disease, already harbour occult distant metastases and display a poor 5-year survival rate. Squamous cell carcinoma of the urinary bladder (SCCUB) is a variant histolog- ical pattern of urothelial carcinoma. Pure SCCUB is secondary to urothelial changes resulted from chronic schistosomiasis haematobium, and prolonged mucosal irritation from long standing indwelling catheter in paraplegic patients. Pure SCCUB has a better survival compared to mixed tumor and/or muscle invasive urothelial carcinoma (Wishahi et al, 2014). Tumor cells undergo invasion and metastasis as sequence of epithelial-mesenchymal transition (EMT), a process by which cells acquire motility, and overriding senescence which is an endogenous defence mechanism against tumor progression, oncogenic activation of Twist 2 proved essential for EMT and senescence. Over expression of Twist 2 was found in colon cancer, cervical cancer, and breast cancer. EMT comprises the processes by which cells 
transit between epithelial and mesenchymal states, and they play integral roles in cancer metastasis (Heerboth et al, 2015). Metastasis is the main cause of death from bladder cancer, and micro metastasis, as the first step of tumor metastasis, appears to be extremely important, EMT is a key step during embryonic development and epithelial tumor metastasis EMT causes changes in cell-cell and cell-extracellular matrix interactions resulting in the transmigration of cancer cells, thus leading to metastasis (Wang et al, 2014), EMT changes occurred at the molecular level before changes in cancer morphology was observed (Zhao et al, 2014). The EMT regulatory proteins Slug and Twist are unregulated in human bladder carcinoma such expression levels may contribute to the progression of tumors in bladder cancer, the potential role of Twist 2 as the prognostic factor in bladder cancer was identified. Twist expression could be used as a predictive factor in bladder cancer but also as a novel target for clinical therapy ( $\underline{\mathrm{Yu}}$ et al, 2010).

The study aimed to assess the expression of Twist 2 in squamous cell carcinoma to elucidate its low potential for distant metastasis.

\section{Patients Material and Methods}

A retrospective cohort study was done on 87 patients their archival materials included clinical data at initial diagnosis that includes metastatic work up, follow up data for 5 to 10 years, overall survival (OS), Disease free survival (DFS), pathological stage of the tumor pTNM, haematoxylin and eosin stain of the tumor tissue, immunohistochemistry stain for Twist2. Ethical committee agreement was considered on the use of archival materials and clinical data of patient given their informed consent at the time of admission to the hospital. Between January 2010 and May 2015, 87 consecutive patients having squamous cell carcinoma (SCC) of the urinary bladder (UB) underwent radical cystectomy, with limited pelvic lymph node dissection beginning at the bifurcation of the common iliac vessels, including the external iliac vessels and obturator fossa. The cystectomy procedure remained unchanged throughout this period and one surgeon performed all procedures. Urinary diversion was orthotopic ileal neobladder, ileal conduit, or uretero cutaneous urinary diversion depending on ureteric dilation.Patients did not receive adjuvant chemotherapy or radiotherapy. Mean age was 52 years (range 4772), they were 70 men and 17 women. Patients were identified in two groups, group A) comprised 65 with stage pT2 N0 M0, group B) comprised 22 patients with stage pT3-4a No Mo. Ten patients with muscle invasive urothelial carcinoma were enrolled as positive control for Twist and FGFR3 immunostaining, 10 patient with no bladder carcinoma or other malignancies were negative control.

Immunohistochemistry for Twist2: formalin fixed Paraffin-embedded sections of $4 \mathrm{~mm}$ thickness were stained with Hematoxylin and eosin (H\&E) stain to evaluate the initial tissue diagnosis of SCC of the Urinary bladder. Paraffin-embedded sections of $4 \mathrm{~mm}$ thickness were processed using Anti-Twist2 concentrated antibody (Abcam, ab 57997, USA), antigen retrieval was performed in all cases by steam-heating the slides in $1 \mathrm{mmol} / \mathrm{l}$ solution $(\mathrm{pH} 9.0)$ for $45 \mathrm{~min}$. After blocking of endogenous biotin, staining was performed using an automated immunostainer (UltraBenchmark, Ventana, USA), followed by detection using Ultraview detection Kit (Ventana, USA). Positive and negative control sections were used for each assay. For quantification of immunological reactions, Twist2 expression was classified as negative, weak, moderate, and over-expression (Fig.1), the twist2 over expression denoted dense staining of both cytoplasm and nucleus. Negative external control staining was done by omitting primary antibodies and well known cases having no bladder cancer or other malignancies, Positive controls Twist2 and FGFR32 were a known muscle invasive urothelial 
tumor, different types of Twist 2 and FGFR3 expression are shown in (Fig. 1). The acquisition of images was done with Nikon Eclipse E600 and software program Lucia 5. Positive controls Twist2 2 were a known muscle-invasive urothelial tumor; fraction of positively stained tumor cells was scored semi-quantitatively after examining 5 high power fields for each case. Clinical evaluation was done every 6 months with laboratory and imaging studies for local recurrence and distant metastases, statistical analysis were mean, range, and percentage.

\section{Results}

The median follow-up was 7.7 years for all surviving patients (70 men \&17 women) with ages 47-72 years (mean 52). They were identified in two groups: A: comprised 65 with stage pT2 N0 M0, and B: comprised 22 patients with stage pT3-4a N0 M0. The retrospective cohort included 87 patients with SCC of UB, and 10 patients as a control group, and another 10 patients with muscle invasive urothelial carcinoma of urinary bladder. Total cohort was 107 patients 65/87 were stage T2 N0 Mo and 22/87 were stage pT3-4a N0 M0. The 10 negative control patients neither had bladder cancer nor other malignancies, and another 10 positive control ones with muscle. Invasive urothelial bladder carcinoma as the high expression of
TWIST2 were not tested for survival.

The median follow-up was 7.7 years for all surviving patients. The 7.7 and years actuarial overall survival rate for Stage T2 N0 M0, $89 \%$ and 13\%; Stage T3-T4a N0 M0 was $72 \%$ \& $22.7 \%$ (Tab. 1). Only cytoplasmic staining was observed, negative Twist 2 expression was observed in 36 cases, faint in zero intensity was observed in 33 cases indicating low expression, medium staining for Twist 2 was found in 16 cases. Degrees of Twist 2 expression were noted in corresponding positive controls and absence of staining was noted in corresponding negative controls (Fig. 1). Overall survival in urinary bladder Squamous cell carcinoma (SCC) of pT2 N0 M0 and pT3-4a N0 M0 that showed negative, low, or moderate Twist 2 expression on immunohisto-chemis-try staining correlated with survival for 5-7.7 years after radical cystectomy $(\mathrm{N}=87)$. Patients with pT2 N0 M0 with negative or weak twist 2 expression had a high overall survival of 7.7 years that was $89 \%$ \& 5 years survival was $11 \%$. Patients with higher tumour stage of pT3-4a No Mo survived for 7.7 years \& 5 years of $72.75 \& 22.75$ respectively. Positive Twist 2 expression of cytoplasmic and nuclear staining was not found in SCC of the urinary bladder.

Table 1:Overall survival in urinary bladder SCC of pT2 N0 M0 and pT3-4a N0 M0 shat showed negative, low, or moderate Twist 2 expression on immunohistochemistry staining correlated with survival for 5-7.7 years after radical cystectomy $(\mathrm{N}=87)$

\begin{tabular}{|c|c|c|c|}
\hline $\begin{array}{c}\text { Survival } \\
\text { time }\end{array}$ & $\begin{array}{c}\text { Bladder SCC } \\
\text { pT2 N0 M0 } \\
(\mathrm{n}=65)\end{array}$ & $\begin{array}{c}\text { Bladder SCC } \\
\text { pT3-4a N0 Mo } \\
(\mathrm{n}=22)\end{array}$ & $\begin{array}{c}\text { TWIST2 expression in initial diagno- } \\
\text { sis bladder SCC } \\
\text { Negative/ weak /moderate/ strong }\end{array}$ \\
\hline 7.7 years & $58(89 \%)$ & $16(72.7 \%)$ & $\begin{array}{c}\text { Negative }(\mathrm{n}=36), \text { weak }(\mathrm{n}=33), \text { medi- } \\
\text { um }(16), \text { strong }(\mathrm{n}=0)\end{array}$ \\
\hline 5 years & $13(11 \%)$ & $5(22.7 \%)$ & \\
\hline
\end{tabular}

\section{Discussion}

Generally speaking, Bladder cancer is the most common male malignancy in Egypt, consists predominantly of urothelial cell carcinoma (UCC) and squamous cell carcinoma (SCC), and disparities in incidence exist between men and women regardless of geographic region. The SCC of the urinary bladder was not common in western world, the common bladder tumor is the urothelial carcinoma whether non-muscle invasive or muscle invasive type, muscle invasive urothelial carcinoma has a high potential for recurrence and metastasis (Yu et al, 2010).

Goerlitz et al. (2011) in Egypt suggested that common genetic variations in GSTM1, GSTT1, and GPX1 were not associated with bladder cancer risk overall and that the well- 
known environmental risk factors, such as smoking and schistosomiasis haematobium and did not interact with these genes to modulate risk. The present study showed a good Os and DFS in organ confined SCCUB ranged from $72.7 \%-89 \%$ in 7.7 years survival. Good Os and DFS correlated with Twist 2 expression at initial diagnosis at time if cystectomy, immunohistochemical staining of Twist 2 showed down-regulation in the form of negative or low expression that were in $79.3 \%$, while medium expression $19.7 \%$ without over-expression.

The present data were contrary to the urothelial carcinoma that exhibited strong expression of Twist 2 potential for the recurrence and metastasis (Waller et al, 2010; Yu et al, 2010; Zhao et al, 2014).

\section{Conclusion}

The outcome results showed that the Twist 2 expression in squamous cell carcinoma of the bladder in conjunction with the other reports on urothelial carcinoma of the bladder down regulation of Twist 2 indicated the good survival in organ confined bladder cancer.

Twist2 would be used as a biological marker to predict metastasis and tumor progression Twist 2 biomarker and tumor pathologic stage of the squamous cell carcinoma of the urinary bladder have a synergistic role in the biologic behaviour of bladder SCC, negative or low Twist 2 expression denoted good Os and DFS. Twist 2 oncogenic biological marker in urothelial carcinoma of the bladder with a reverse expression to evaluate tumor may improve prognostic models and identify patients who might benefit from adjuvant therapies and/or target therapy.

\section{References}

Goerlitz, D, El Daly, M, Abdel-Hamid, M, Saleh, DA, Goldman, L, et al, 2011: GSTM1, GSTT1 null variants, and GPX1 single nucleotide polymorphism are not associated with bladder cancer risk in Egypt. Cancer Epidemiol. Biomarkers Prev. 20, 7:1552-4.

Heerboth, S, Housman, G, Leary, M, Byler, S, Lapinska, K, et al, 2015: EMT and tumor metastasis. Clin. Translat. Med. 4:6-12

Li, HT, Duymich, CE, Weisenberger, DJ, Liang, G, 2016: Genetic and Epigenetic Alterations in Bladder Cancer. Int. Neurourol. J. 20, 2:S8494. Review.

Waller, H, Robert, G, Pasticier, G, Ravaud, A, Ballanger, P, Reiter RE, et al, 2010: The epithelial-mesenchymal transition-inducing factor TWIST is an attractive target in advanced and/or metastatic bladder and prostate cancers. Urol. Oncol. 28: 473-9.

Wang, T, Li, Y, Wang, W, Tuerhanjiang, A, Wu, Z, et al, 2014: Twist2, the key Twist isoform related to prognosis, promotes invasion of cervical cancer by inducing epithelial-mesenchymal transition and blocking senescence. Hum. Pathol. 45, 9:1839-46

Wishahi, M, Elganzoury, H, Elhouly, A, Badawi, M, 2014: Urothelial carcinoma of the urinary bladder mixed with squamous differentiation or squamous cell carcinoma in areas with schistosomiasis is showing high risk of recurrence and poor survival. J. Egypt. Soc. Parasitol. 44, 2:467-73.

Yu, Q, Zhang, K, Wang, X, Liu, X, Zhang, Z, 2010: Expression of transcription factors snail, slug, and twist in human bladder carcinoma. J. Exp. Clin. Cancer Res. 29, 1:119-26

Zhao, J, Dong, D, Sun, L, Zhang, G, Sun, L, 2014: Prognostic significance of the epithelialto-mesenchymal transition markers e-cadherin, vimentin and twist in bladder cancer. Int. Braz. J. Urol. 40, 2:179-89.

\section{Explanation of Figure}

Fig.1: Representative microscopic images of immunohistochemical staining for TWIST2 in urinary bladder squamous cell carcinoma (SCC, $\times 40$ ). A- No immunohistochemical staining indicating absence of Twist 2 expression in urinary bladder SCC. B- Faint (weak) immunohistochemical staining indicating low expression of Twist2 in SCC of urinary bladder. C- Medium immunohistochemical staining indicating low expression of Twist 2 in SCC of urinary bladder. D- Positive model for Strong immunohistochemical staining indicating high Twist 2 expression in a case of muscle-invasive urothelial carcinoma of urinary bladder. Twist 2 expression was determined immunohistochemically using anti-Twist2 antibodies in a cohort of urinary bladder $\operatorname{SCC}(n=87)$. 


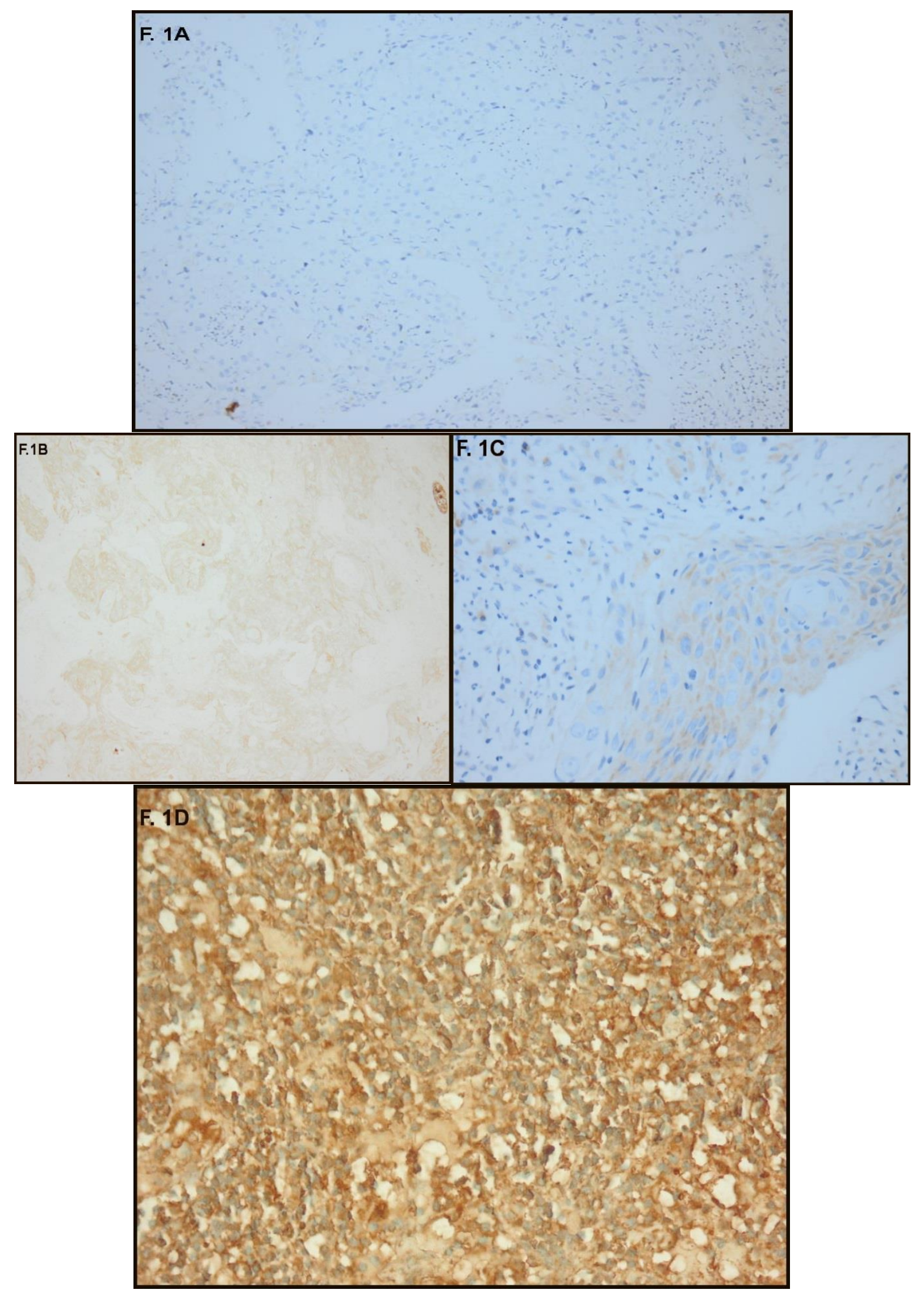

